\title{
Lo que el pediatra de Atención Primaria debería saber sobre el sueño
}

\author{
G. Pin Arboledas ${ }^{\mathrm{a}}$, R. Ugarte Libano ${ }^{\mathrm{b}}$ \\ aUnidad Valenciana del Sueño. Hospital Quirón. Valencia. España. \\ bCS Olaguibel. Servicio Vasco de Salud-Osakidetza. Vitoria-Gasteiz. España.
}

\begin{abstract}
Resumen
El papel del pediatra de Atención Primaria es fundamental en el diagnóstico precoz y el tratamiento de los principales trastornos del sueño. Se repasan las diez principales preguntas que un pediatra de Atención Primaria se debe plantear, y se concluye que las que resumen las funciones del pediatra en cuanto a la educación para la salud en medicina del sueño son: realizar el correcto diagnóstico de los eventos fisiológicos y normales relacionados con el sueño, establecer las medidas de prevención o de promoción de los trastornos del sueño, realizar en cada examen de salud la anamnesis en la que se incluyen los test de cribado, que son los elementos base de la prevención y del diagnóstico precoz, e iniciar un tratamiento precoz de los problemas el sueño.
\end{abstract}

Palabras clave: Sueño. Prevención. Educación. Atención Primaria.

\section{What Primary Care pediatricians should know about sleep}

\section{Abstract}

The role of the Primary Care pediatrician is essential for early diagnosis and treatment of major sleep disorders. We review the top ten questions that a Primary Care pediatrician should ask himself. A summary on the roles of the pediatrician in terms of health education and treatment in sleep medicine is drawn: to make the correct diagnosis, to know normal physiological events associated with sleep, to establish preventive measures, to screen out sleep disorders in each health examination, which includes screening tests that are the basic elements of prevention and early diagnosis, and to initiate an early treatment of the sleep problems.

Key words: Sleep. Prevention. Health education. Primary Health Care.

\section{Introducción}

El decálogo que el pediatra de Atención Primaria debería ser capaz de res- ponder en referencia a la medicina del sueño podríamos resumirlo en:

1. ¿Cuál es la fisiología básica del sueño?

Ramón Ugarte Libano: rugarte@gmail.com

Los autores declaran no presentar conflictos de intereses en relación con la preparación y publicación de este artículo. 
2. ¿Cómo evoluciona el dormir a lo largo de la etapa pediátrica?

3. ¿Qué problemas relacionados con el sueño puede tener la población que atiendo?

4. ¿Qué clínica me puede indicar que estoy ante un trastorno del sueño?

5. ¿De qué herramientas dispongo en la consulta para realizar una correcta aproximación a los problemas del sueño?

6. ¿Qué herramientas terapéuticas tengo a mi disposición en Atención Primaria?

7. ¿Existe una población con mayor riesgo de tener problemas con el sueño?

8. ¿Cuando debería derivar a un paciente a una Unidad de Sueño?

9. ¿Debo establecer medidas de seguimiento a los pacientes ya tratados? Si es así, ¿cuáles deberían ser?

10. Qué medidas preventivas de los problemas con el sueño puedo aplicar en la consulta.

\section{¿Cuál es la fisiología básica del sueño?}

El sueño se divide en dos estados diferenciados: sueño NREM (de ondas lentas) y sueño REM o de movimientos oculares rápidos. El sueño NREM, a su vez, se divide en tres fases (1, 2 y 3 ) que van de menos a más profundidad del sueño. Los estados REM y NREM se alternan en ciclos que en el Recién Nacido duran 40 minutos y se van alargando hasta los 90 minutos del adulto.

Durante el sueño, se producen una serie de cambios en las secreciones hormonales, entre los que podemos destacar':

- Hormona del crecimiento: se segrega principalmente en la primera fase de sueño lento.

- Prolactina: su secreción se incrementa a los 60-90 minutos.

- Testosterona: se observan los niveles más altos.

- Hormona tiroestimulante: alcanza su máximo por la tarde y durante el sueño.

- Cortisol: su secreción es inhibida.

- Melatonina: su nivel empieza a incrementarse por la tarde, y alcanza el máximo entre las tres y las cinco de la madrugada.

- Aldosterona: alcanza su máximo antes del despertar.

- Renina: descenso marcado durante el sueño REM (desciende la filtración glomerular).

\section{¿Cómo evoluciona el dormir a lo} largo de la etapa pediátrica? ?2-4 $^{-2}$

- Evolución del sueño de uno a tres meses de vida: 
- El bebé se duerme en sueño activo, por lo que los movimientos, gestos y emisión de sonidos al iniciar el sueño son la norma.

- El tiempo de vigilia durante la noche es de alrededor del $6 \%$ del tiempo total de sueño.

- Evolución del sueño de los tres a los 12 meses de edad:

- De los cuatro a los seis meses se consolida el largo periodo de sueño nocturno, se establece el ritmo de temperatura. Se desarrolla un ritmo "multimodal" caracterizado por una siesta en la mañana, siesta en la tarde, y largo sueño nocturno.

- Número medio de despertares: aproximadamente nueve al mes de edad y 4,5 al año de edad.

- Evolución del sueño del primer al segundo año de vida

- Año y medio de edad: se retira la siesta matinal, aparece un ritmo "bimodal" con una siesta a medio día.

- El sueño total es de 14-15 horas y se consolida un largo periodo de sueño nocturno de 10 a 12 horas.

- La latencia de sueño: 15-30 minutos.

- El porcentaje de niños que duermen cinco horas seguidas duran- te la noche se va incrementando: $70 \%$ a los tres meses, $80 \%$ a los seis meses y $90 \%$ a los 12 meses.

- Evolución del sueño de los dos a los cinco años de edad ${ }^{6,7}$ :

- Periodo nocturno de aproximadamente diez horas.

- Durante los 2-4 primeros años continúan las siestas diurnas cortas: $25 \%$ de niños de dos años tienen dos siestas al día, el 66\% una única siesta y el $8 \%$ tienen siestas de manera irregular.

- Un 10\% de los niños duerme más de 12 horas y un 10\% menos de 9,5 horas.

- La disminución del tiempo total de sueño durante este periodo es de 20 minutos por año de edad.

- A los dos años de edad, se despierta el $20 \%$ de los niños, cifra que se reduce al $14 \%$ a los tres años.

- El sueño de los adolescentes:

- Tendencia biológica al retraso de fase con dificultad para dormirse y levantarse pronto; por ello, el problema más frecuente es la excesiva somnolencia diurna9. Los estudiantes que cambian su horario de sueño más de dos horas pueden experimentar alteraciones en la atención, concentra- 
ción, razonamiento y habilidades psicomotoras ${ }^{10}$.

- Se ha demostrado que, incluso a estas edades, aumentar el conocimiento de los adolescentes sobre la higiene del sueño incrementa las prácticas saludables de sueño.

En la página Web de la AEPap están disponibles las gráficas con los percenti- les de las horas habituales de sueño según la edad del niño.

\section{¿Qué problemas relacionados con el sueño puede tener} la población que atiendo?

Podríamos resumir las alteraciones más frecuentes en la infancia modificando el esquema de Carolyn Thierdke (Am Fam Physician. 2001;63:277-84) (tabla 1).

\begin{tabular}{|c|c|c|}
\hline Edad & Problema & Intervención terapéutica \\
\hline $0-4$ meses & $\begin{array}{l}\text { Los despertares y } \\
\text { alimentaciones nocturnas son } \\
\text { un hito normal en el desarrollo }\end{array}$ & Información sanitaria \\
\hline 4-12 meses & $\begin{array}{l}\text { Despertares nocturnos } \\
\text { Rechazo a acostarse } \\
\text { Compartir cama } \\
\text { Alimentación nocturna }\end{array}$ & $\begin{array}{l}\text { Extinción-despertares programados } \\
\text { Ritual del presueño } \\
\text { Potenciar un "apego seguro" } \\
\text { Alargar el tiempo de espera alimenticio + disminuir } \\
\text { progresivamente el volumen/duración de la toma }\end{array}$ \\
\hline 2-4 años & $\begin{array}{l}\text { Insomnio de inicio y/o } \\
\text { mantenimiento }\end{array}$ & $\begin{array}{l}\text { Ritual del presueño claro-objeto transicional- } \\
\text { refuerzos positivos-límites claros-adecuar el } \\
\text { momento de acostar } \\
\text { Potenciar un "apego seguro" }\end{array}$ \\
\hline 3-8 años & $\begin{array}{l}\text { Terror nocturno } \\
\text { Pesadillas }\end{array}$ & $\begin{array}{l}\text { Información-evitar refuerzos o beneficios-no } \\
\text { despertar-despertares conductuales-en caso } \\
\text { necesario medicación } \\
\text { Controlar juegos-vídeos-televisión } \\
\text { Consolar } \\
\text { Evitar refuerzos o beneficios }\end{array}$ \\
\hline 6-12 años & Enuresis nocturna & $\begin{array}{l}\text { Alarma-refuerzos positivos-práctica positiva } \\
\text { Medicación (¿?) }\end{array}$ \\
\hline 8-12 años & $\begin{array}{l}\text { Sonambulismo } \\
\text { Soliloquia }\end{array}$ & Medidas de seguridad-despertar programado \\
\hline Adolescencia & $\begin{array}{l}\text { Síndrome de retraso de fase } \\
\text { Narcolepsia } \\
\text { Excesiva somnolencia }\end{array}$ & $\begin{array}{l}\text { Límites claros-educación-horarios claros } \\
\text { Remisión }\end{array}$ \\
\hline Cualquier edad & SAHS & Remisión \\
\hline
\end{tabular}

SAHS: síndrome de apnea e hipopnea del sueño. 
¿Qué clínica me puede indicar que estoy ante un trastorno del sueño?

Los síntomas de sospecha de problemas con el sueño son ${ }^{11-13}$ :

- Síntomas mayores:

- En niños mayores de un año: presencia de tres a cinco requerimientos de acción durante la noche, más de tres noches a la semana.

- Sospecha de somnolencia diurna excesiva en un niño de cinco años.

- Dificultad creciente de despertarlo por las mañanas.

- Estado de excesiva irritación al despertar.

- Latencia de sueño mayor de media hora de manera habitual.

- Síntomas menores de sospecha:

- Dificultades de concentración y memoria sin otra causa médica.

- Deterioro del rendimiento escolar.

- Incremento de irritabilidad o aparición de conductas agresivas.

- Aumento espontáneo y voluntario del tiempo de sueño acompañado de mejoría de la conducta.

Por todo ello, es importante que el pediatra de Atención Primaria:

- Pregunte sobre el sueño.

- Domine varias modalidades de abordaje de los problemas del sueño.
- Comprenda que lo que es correcto y adecuado para una familia puede no serlo para otra.

- Sea consciente de que las necesidades de sueño varían a lo largo de la infancia.

- Tenga en cuenta que los problemas del sueño pueden manifestarse solo durante el día.

- Sepa que los autorregistros o agendas de sueño son muy útiles y ahorran tiempo de consulta.

Un grupo de preguntas puede orientar a la presencia de problemas:

- Cuando duerme, ¿se despierta al menos tres noches a la semana más de tres veces por la noche?

- ¿Ronca o hace ruido al respirar mientras duerme?

- ¿Cuantas horas duerme cada día?

- ¿Tiene conductas o movimientos que le llamen la atención mientras duerme?

¿De qué herramientas dispongo en la consulta para realizar una correcta aproximación a los problemas del sueño?

La validez de las encuestas subjetivas para reflejar las características del sueño está demostrada ${ }^{14}$. En la página Web de la AEPap están disponibles los cuestionarios utilizados generalmente. 
¿Qué herramientas terapéuticas tengo a mi disposición en Atención Primaria?

La Academia Americana de Medicina del sueño indica ${ }^{15}$ :

- La educación de los padres y la prevención son terapias recomendables en el tratamiento de los problemas al acostarse y los despertares nocturnos. Estándar. Evidencia: I-II.

- La extinción no modificada y la extinción de una conducta no deseada con la presencia paterna son terapias eficaces y recomendables. Estándar. Evidencia: I-II.

- La extinción gradual de una conducta no deseada es una terapia efectiva y recomendable en el tratamiento de los problemas al acostarse y despertares nocturnos. Guía. Nivel de evidencia: IIIII.

- El retraso de la hora de acostarse y las rutinas positivas son terapias efectivas y recomendables en el tratamiento de los problemas al acostarse y despertares nocturnos. Guía. Nivel de evidencia: II-III.

- El uso de los despertares programados es una terapia efectiva y recomendable en el tratamiento de los problemas al acostarse y des- pertares nocturnos. Guía. Nivel de evidencia: II-III.

- No hay suficiente evidencia para recomendar una combinación o intervenciones mixtas en el tratamiento de los problemas al acostarse y despertares nocturnos.

Los ensayos clínicos que valoran el tratamiento farmacológico del insomnio en un niño sin complicaciones no encontraron diferencias significativas en la evolución cuando se mide a largo plazo, si bien no se tuvo en cuenta el uso de la melatonina ${ }^{16}$.

El tratamiento farmacológico se debe basar en un diagnóstico y no en síntomas ni en las expectativas parentales, en el contexto de un amplio plan de tratamiento y en combinación con estrategias no farmacológicas y mejoría de la higiene del sueño. Debe tener un objetivo realista, claramente definido y discutido con la familia así como un programa de seguimiento. Las indicaciones de un tratamiento farmacológico del insomnio infantil en un niño por otra parte sano serían ${ }^{17}$ : seguridad y bienestar del niño en peligro; padres incapaces de instaurar medidas no farmacológicas, junto con medidas de higiene y conductuales; insomnio en el contexto de una enfermedad médica, una situación estresante o un viaje. 
¿Existe una población con mayor riesgo de tener problemas con el sueño?

Disponemos de suficiente evidencia científica que nos permite relacionar como población de riesgo de presentar diferentes alteraciones del sueño $a$, por ejemplo:

- Alteraciones del estado ponderal (obesidad-sobrepeso, desnutrición) ${ }^{18-20}$.

- Trastorno de hiperactividad con déficit de atención o sin él ${ }^{21-22}$.

- Deterioro de su rendimiento escolar no explicable por otras causas ${ }^{23}$.

- Hipertrofia del anillo de Waldeyer y malformaciones craneofaciales ${ }^{24}$.

- Síndrome de Down ${ }^{25}$.

\section{¿Cuándo debería derivar a un} paciente a una Unidad de Sueño?

En general, se debería plantear la derivación a una Unidad de Sueño en los siguientes $\operatorname{casos}^{26}$ :

- Problemas a la hora de acostarse que no responden a medidas conductuales simples o que son muy intensas y crean niveles elevados de alteración familiar.

- Parasomnias con síntomas más allá de la alteración el sueño.

- Cuadros mórbidos asociados que exigen un tratamiento específico.
- Alteraciones del ritmo circadiano.

- Síntomas de excesiva somnolencia diurna sin déficit de sueño.

- Alteraciones de la respiración durante el sueño:

- Se remitirá de manera ordinaria: la presencia de síntomas claros de trastorno respiratorio en un niño por otra parte sano con una conducta adecuada y buen rendimiento escolar.

- Se remitirá de manera preferente: la presencia de riesgos adicionales y/o de síntomas/signos de gravedad.

\section{¿Debo establecer medidas}

de seguimiento a los pacientes

ya tratados? Si es así, ¿cuáles deberían ser?

En general, los problemas conductuales del sueño no requieren un seguimiento especial. Sin embargo, en cuanto a los trastornos respiratorios, aproximadamente el $20 \%$ necesitan reevaluarse ${ }^{27}$.

\section{¿Qué medidas preventivas} de los problemas con el sueño puedo aplicar en la consulta?

Se han establecido medidas preventivas en función de la etapa del niño ${ }^{28}$ :

- Del recién nacido a los dos meses de edad: 
- Los niños tienen que aprender a dormirse. Cada niño marca su propio ritmo para dormir.

- Hasta los dos meses de edad, después de comer el niño inicia lo que llamamos "sueño activo" (el niño parece inquieto), este tipo de sueño suele durar unos 30-40 minutos hasta que entra en un sueño más profundo, que denominamos "sueño tranquilo".

- Procure que su bebé permanezca despierto mientras come. Si se duerme, estimúlelo para que se despierte antes de depositarlo en la cuna. Después de la alimentación, colocaremos al bebé en la cuna, mientras está todavía despierto.

- Los padres deben permanecer con el bebé para calmarlo y disminuir su activación pero no para dormirlo.

- A partir de la instauración de la lactancia materna se puede utilizar el chupete.

- Es muy recomendable que la madre siga los mismos horarios de sueño que el niño.

- Los padres deben facilitar un horario y un lugar adecuado para el sueño. Deben calmar y preparar al bebé para el sueño pero NO tienen que dormirlo.
- Al cumplir los dos meses

- Hacer siempre lo mismo a la hora de acostarlo.

- No despierte al bebé para alimentarlo.

- Al cumplir los seis meses:

- Durante los primeros ocho meses puede ser habitual y normal que el bebé se despierte por la noche: NO lo alimente, NO encienda la luz y NO lo saque de la cuna. Consuélelo con caricias y frases cariñosas.

- Busque un peluche que sea su compañero en la cuna.

- Deje abierta la puerta del cuarto.

- Al cumplir los 12 meses:

- El ambiente debe ser tranquilo y oscuro.

- La hora diaria de levantarse debe ser aproximadamente la misma todos los días.

- La hora diaria de acostarse debe ser aproximadamente la misma todos los días.

- La temperatura de la habitación debe ser confortable: un exceso de calor o frío favorece el despertar nocturno.

- El ruido ambiental será el mínimo posible.

- Evitar acostarlo con hambre. Sin embargo, el exceso de líqui- 
dos favorece el despertar nocturno.

- Debe aprender a dormirse solo, sin ayuda.

- Debe transmitir el mensaje de que se le está enseñando a dormir, de manera autónoma, y que no se trata de ningún castigo ni de una disputa entre padres y el niño.

- La actividad física vigorosa se evitará 1-2 horas antes de acostarlo.

- Evitar alimentos como el chocolate y bebidas como las colas, café o té.

- Evitar las siestas muy prolongadas o tardías.

- Al cumplir los 18 meses:

- Cuando el niño le esté "dando la noche" reflexione sobre lo ocurrido durante ese día. No le chille, déle la seguridad de que usted estará para tranquilizarlo si lo necesita.

- No se imponga a sí mismo ninguna hora límite para conseguir que el niño duerma bien.

- No pierda la calma cuando se despierte a media noche. Si ha habido algún cambio reciente en la vida del niño, no espere que duerma profundamente.
- Permitirle que duerma fuera de la cama no le ayudará a aprender a dormirse solo.

- El pecho, el biberón o el chupete parecen muy útiles para dormirse, pero acabará necesitándolos cada vez que tenga que dormirse, y en cuanto se despierte.

- Altérnese con su pareja: descansará más y el niño no "dirigirá" la noche. Si esto no es posible, haga una pausa cuando vea que "pierde los nervios". Lo importante es transmitir tranquilidad y seguridad educativa.

- Cada familia tiene su nivel de tolerancia y sus propias creencias: no hay sistemas buenos ni malos, solo diferentes.

- De los dos a los cinco años de edad:

- Durante el día, es recomendable que los niños duerman la siesta, como complemento al sueño nocturno.

- Cuando de manera esporádica su hijo tenga dificultades para conciliar el sueño, reflexione sobre lo acontecido ese día y no le chille. Debe transmitirle seguridad y tranquilidad.

- No se imponga ninguna hora límite para que se duerma ese día. 
- No pierda la calma.

- A partir de los 11 años (dirigidos al adolescente):

- Debes tener horarios regulares. Incluso los fines de semana.

- Exposición de luz intensa por la mañana. Te ayudará a adelantar la fase de sueño.

- Debes favorecer las actividades estimulantes en horas con tendencia al sueño diurno.

- Debes evitar las actividades de alerta (estudio, videojuego...) una hora antes de dormir.

- No tomar estimulantes (cafeína, bebidas de cola...), especialmente después de la comida del mediodía.

- Regularidad en el horario de las comidas.

- No debes dormirte con la televisión puesta (disminuye la profundidad del sueño). No colocar televisión en la habitación.

- Practicar regularmente ejercicio físico.
- Debes relajarte y expresar adecuadamente tus emociones.

En resumen, las funciones del pediatra en cuanto a la educación para la salud en Medicina del Sueño son:

- Realizar el correcto diagnóstico de los eventos fisiológicos y normales relacionados con el sueño (número de despertares según la edad del niño, duración de la latencia de sueño...) con la debida educación sanitaria de las familias con el objetivo de evitar iatrogenia médica y/o familiar.

- Establecer las medidas de prevención o de promoción de los trastornos del sueño.

- Realizar en cada examen de salud la anamnesis en la que se incluyen los test de cribado que son los elementos base de la prevención y del diagnóstico precoz.

- Tratamiento precoz de los problemas el sueño.

- Formación continuada en Medicina del Sueño.

\section{Bibliografía}

1. Goehler L, Gaykema R. Immune signaling to brain: Mechanisms and potencial pathways influencing sleep. En: Pandi-Perumal SR, Cardinali
DP, Chrousos GP. Neuroimmunology of sleep. New Jork: Springer; 2007. p. 120-31.

2. Gaylor E, Burnham M, Goolin-Jones B, Anders T. A longitudinal follow-up study of young children's slep patterns using a developmental 
classification system. Behav Sleep Med. 2005; 3(1):44-61.

3. Santamaría J. Mecanismos y función del sueño: su importancia clínica. Med Clin (Barc). 2003;120:750-5.

4. Franco P, Kato I, Richardson H, Yang J, Montemitro E, Horne R. Arousal from sleep mechanisms in infants. Sleep Medicine. 2010;11: 603-14.

5. Acebo C, Sadeh A, Seifer R, Tzischinsky O, Hefer A, Craskadon M. Slep/wake pattrerns derived from activity monitoring and maternal report for healthy 1 to 5 year old children. Sleep. 2005;28:1568-77.

6. Spilsbury JC, Storfer-Isser A, Rosen C, Kirchner $L$, Benham $H$, Redline S. Sleep behavior in an Urban US sample of school-aged children. Arch Pediatr Adolesc Med. 2004;158:988-94.

7. Gulliver T, Gozal D. Polysomnographic characteristics in normal preschool and early scholaged children. Pediatrics. 2006;117:741-53.

8. Laberge L, Petit D, Vitaro C, Tremblay R, Momtplaisir J. Development of sleep patterns in early adolescence. J Sleep Res. 2001;10:59-67.

9. García Jimenez MA, Salcedo Aguilar F, Rodríguez Almonacid FM, Redondo Martinez MP, Monterde Aznar ML, Marcos Navarro Al y cols. Prevalencia de los trastornos del sueño en adolescentes de Cuenca, España. Rev Neurol. 2004; 39:18-24.

10. Pagel JF, Forister N, Kwiatkowki C. Adolescent sleep disturbance and school performance: the confounding variable of socioeconomics. J Clin Sleep Med. 2007;3(1):19-23.

11. Sadeh A, Raviv A, Gruber R. Sleep patterns and sleep disruptions in school-aged children. Dev Psicol. 2000;36:291-301.

12. Peli $P$, Touchette E, Tremblay RE, Bolvin $M$, Montplaisir J. Dysomnias and parasomias in early childhood. Pediatrics. 2007; e1016-25.
13. Kotagal S. Parasomnias in chilhood. Sleep Med Rev. 2009;13:157-68.

14. SekineM, ChenX, HamanishiS, WangH, YamagamiT, KagamimoriS. TheValidity of Sleeping Hours of HealthyYoung Children as Reported by Their Parents. J Epidemiol. 2002;12:239-42.

15. American Academy of Sleep Medicine Report. Practice parameters for behavioral treatment of bedtime problems and night wakings in infants and young children. Sleep. 2006;29: 1277-81.

16. Ramchandani P, Wiggs L, Webb V, Stores G. A systematic review of treatments for settling problems and night waking in young children. BMJ. 2000;320:209-13.

17. Owens JA, Andreason P, Babcock D. The use of pharmacotherapy in the treatment of pediatric insomnia in primary care; rational approaches. A consensus meeting summary. J Clin Sleep Med. 2005;1(1):49-59.

18. Peirano $P$, Algarin $C$, Chamorro R, Reyes $S$, Durán S, Garrido $M$ et al. Sleep alterations and iron deficiency anemia in infancy. Sleep Med. 2010:637-42.

19. Nielsen LS, Danielsen KV, Sørensen TI. Short sleep duration as a possible cause of obesity: critical analysis of the epidemiological evidence. Obes Rev. 2010 Mar 24. [Epub ahead of print].

20. Touchette E, Petit D, Tremblay RE, Boivin $M$, Falissard B, Genolini C et al. Associations between sleep duration patterns and overweight /obesity at age 6. Sleep. 2008;31:1507-14.

21. Sung V, Hiscock $H$, Sciberras $E$, Efron D. Sleep Problems in Children With Attention-Deficit/Hyperactivity Disorder. Prevalence and the Effect on the Child and Family. Arch Pediatr Adolesc Med. 2008;162:336-42.

22. Tomás Vila $M$, Miralles Torres $A$, Beseler Soto B, Revert Gomar M, Sala Langa MJ, Uribelarrea Sierra A. Relación entre el trastorno por défi- 
cit de atención e hiperactividad y los trastornos del sueño. Resultados de un estudio epidemiológico en la población escolar de la ciudad de Gandía. An Pediatr (Barc). 2008;69:251-7.

23. Sans Capdevila O, Kheirandish-Gozal L, Dayyat E, Gozal D. Pediatric Obstructive Sleep Apnea Complications, Management, and Long-term Outcomes. Proc Am Thorac Soc. 2008; 5:274-82.

24. Wildhaber $\mathrm{JH}$, Moeller A. Sleep and respiration in children: time to wake up! Swiss Med Wkly. 2007;137:689-94.

25. Carter M, McCaughey E, Annaz D, Hill CM. Sleep problems in a Down syndrome population. Arch Dis Child. 2009;94;308-10.
26. Owens J, Rosen C, Mindell J. Medication Use in the Treatment of Pediatric Insomnia: Results of a Survey of Community-Based Pediatricians. Pediatrics. 2003;111:e628-35.

27. Waters K, Cheng A. Adenotonsillectomy in the context $f$ obstructive slep apnea. Paediatr Respir Rev. 2009;10:25-31.

28. Grupo Pediátrico de la Sociedad Española de Sueño y Grupo de sueño de la sociedad Española de Pediatría Extrahospitalaria y Atención Primaria (SEPEAP). Medidas preventivas de los problemas del sueño desde el nacimiento hasta la adolescencia. Acta Pediatr Esp. 2010;68(4): 167-73. 